\title{
RECORDS IN CONTEXTS: UN NUEVO MODELO PARA LA REPRESENTACIÓN DE LA INFORMACIÓN ARCHIVISTICA EN EL ENTORNO DE LA WEB SEMÁNTICA
}

\author{
Records in contexts: A new model for the \\ representation of archival information in semantic \\ web scenarios
}

\section{Dunia Llanes-Padrón y Manuela Moro-Cabero}

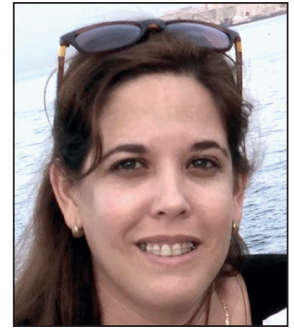

Dunia Llanes-Padrón, doctora en Biblioteconomía y Documentación por la Universidad de Salamanca y posdoctorado en ciencias de la información por la Facultad de Filosofía y Ciencias de la Universidade Estadual Paulista, Brasil, es profesora de la Facultad de Comunicación de la Universidad de La Habana (UH). También ha impartido cursos de pregrado y posgrado en universidades de Brasil, España y Portugal. Miembro del Consejo Nacional de Desarrollo Científico y Tecnológico de Brasil como colaboradora extranjera. Sus líneas de investigación se enfocan a la representación y el acceso a la información archivística, la normalización de la descripción y la gestión documental. http://orcid.org/0000-0001-8639-4706

Universidad de La Habana, Facultad de Comunicación, Departamento de Ciencias de la Información C/ San Pedro entre Ermita y Av. Independencia, Plaza de la Revolución, Edif. Bohemia, La Habana, Cuba duniallp@yahoo.es

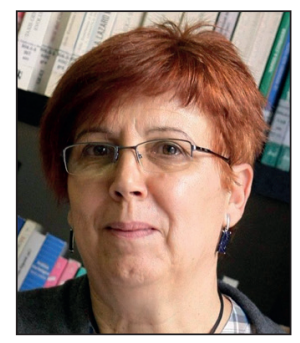

Manuela Moro-Cabero es profesora titular del Departamento de Biblioteconomía y Documentación de la Universidad de Salamanca y profesora invitada en el Curso de pos-graduación de la Facultad de Filosofía y Ciencias de la Universidade Estadual Paulista, Brasil. Es miembro del Grupo de Investigación Ged/A-Gestão Eletrônica de Documentos Arquivísticos, del Conselho Nacional de Desenvolvimento Científico e Tecnológico (CNPq), Brasil, y del Comité Técnico Nacional 50 sobre Información y Documentación de la Asociación Española de Normalización y Certificación (Aenor). Sus publicaciones recientes tratan de la difusión de las normas en España, en particular de la serie Management system standards (MSS) de la ISO. Es miembro del Observatorio de Prospectiva Archivística y Sociedad de la Universidad Carlos III de Madrid.

http://orcid.org/0000-0001-5301-1924

Universidad de Salamanca, Facultad de Traducción y Documentación, Departamento de Biblioteconomía y Documentación Francisco Vitoria, 6-16. 37008 Salamanca, España moroca@usal.es

\section{Resumen}

Se analiza el borrador de Records in contexts $(R i C)$, la propuesta de nuevo modelo conceptual de descripción archivística del Internacional Council of Archives (ICA), y se describen sus principios conceptuales, propiedades, atributos y relaciones. El modelo presenta un diseño novedoso para la representación, acceso, uso, preservación e interoperabilidad de la información archivística en el contexto de la web semántica. El análisis permite comprender las ventajas que ofrece RiC para la integración y compatibilidad de los sistemas de información de archivos con los de bibliotecas, museos y otras instituciones culturales. Se muestra una visión general del modelo, subrayando su importancia para la integración, distribución, reutilización y uso abierto de los recursos archivísticos en entornos digitales.

\section{Palabras clave}

Descripción archivística; Representación de información; Normalización de la descripción; Modelos conceptuales de descripción; Records in contexts; Interoperabilidad semántica.

\section{Abstract}

This article studies the main concepts, properties, attributes, and connections included in Records in contexts (RiC), a draft of a new conceptual model for record description by the International Council of Archives (ICA). The analysis shows the new design of representation, access, use, preservation, and interoperability for information-records that this model represents in the environment of the semantic web. The intrinsic advantages of RiC for the integration and compatibility of records' 
information systems with libraries, museums, and other cultural institutions are shown, also highlighting its relevance for distribution, reutilization, and free-use of records' resources in digital scenarios.

\section{Keywords}

Record description; Representation of information; Standardization of description; Conceptual models for description; Records in contexts; Semantic interoperability.

Llanes-Padrón, Dunia; Moro-Cabero, Manuela (2017). “Records in contexts: Un nuevo modelo para la representación de la información archivística en el entorno de la web semántica”. El profesional de la información, v. 26, n. 3, pp. 525-533.

https://doi.org/10.3145/epi.2017.may.19

\section{Introducción}

Durante los últimos tiempos, la descripción archivística se ha visto revolucionada por los nuevos paradigmas de la ciencia de la información. La descripción, basada en la representación exacta de entidades archivísticas, juega un papel fundamental en la recuperación, el acceso y uso de la información pero, también, es determinante para facilitar la interoperabilidad semántica y la reutilización de los datos archivísticos entre sistemas diversos de información, publicados en ambientes web. Asimismo, la descripción garantiza la gestión, la autenticidad, la veracidad y la preservación de los records archivísticos digitales.

La década de los 90 del pasado siglo se puede considerar como el epicentro de los cambios realizados en el proceso. El Internacional Council of Archives publicó las primeras normas internacionales que iban a guiar el desarrollo homogéneo de la descripción en todas las comunidades archivísticas. ISAD(G), ISAAR(CPF), ISDF e ISDIAH fueron los estándares pioneros del proceso normativo desplegado en esta área. Posteriormente, la archivística se vio inundada de un océano de abreviaturas que simbolizan los estándares publicados en cada país:

- Gran Bretaña, Irlanda, EUA y Canadá cuentan con las normas MAD3, IGAD, DACS, RAD2 y Rcppcn.

- Portugal y Brasil publican ODA y Nobrade respectivamente.

- En España se publicaron las sucesivas normas nacionales y regionales MDM, NEDA-I, Nodac, Nogada, Nepan y Aranor.

- Uruguay desarrolla la Norma uruguaya de descripción archivística (NUDA), a la que se articulan pautas de codificación como EAD y EAC.

A partir de este oleaje normativo se comienza a repensar la conceptualización del proceso. Con ISAD(G) aparece la descripción multinivel, principio con el cual se describen y se relacionan jerárquicamente todas las partes de un fondo. Esta nueva forma de representación basada en la jerarquía de los niveles de organización es conocida, igualmente, como descripción unidimensional pues sólo comprende las relaciones entre los subtipos de una entidad: el documento. Con el tiempo se abordan posturas teóricas que diseñan modelos conceptuales más completos, orientados a una descripción que contemple todas las entidades que intervienen en la producción, gestión y conservación de los documentos. Este método se conoce como descripción pluridimensional pues contiene representaciones y relaciones entre varias entidades archivísticas.

Los modelos conceptuales son un componente importante para lograr la interoperabilidad semántica pues establecen las bases para armonizar datos y sus relaciones lógicas. Los modelos constituyen el antecedente para el desarrollo de ontologías basadas en esquemas de datos para la web (RDF). Asimismo, facilitan la interrelación y la integración de los contenidos archivísticos en internet.

El trabajo que se presenta tiene como objetivo analizar $R e$ cords in contexts. A conceptual model for archival description (RiC-CM) publicado en versión borrador por el ICA. Para ello se realiza un estudio de los principios teóricos, propósitos, entidades, propiedades y relaciones de RiC-CM. Este ensayo es de naturaleza descriptiva y tiene como intención subrayar la importancia de este modelo para la representación, el acceso, uso, preservación e interoperabilidad de la información archivística en el contexto de la web semántica.

La representación de la información archivística juega un papel determinante en la gestión y la reutilización de datos

\section{Records in contexts: Principios teóricos y propósitos}

En 2012 el Internacional Council of Archives formó el Grupo de Expertos en Descripción Archivística (EGAD) sucesor del Comité de Buenas Prácticas y Normas responsable, hasta ese momento, de la normalización. El nuevo grupo tenía dentro de sus objetivos desarrollar un modelo conceptual internacional para la descripción archivística que garantizara la integración e interrelación de las cuatro normas de descripción en sistemas unificados de información.

Durante cuatro años los expertos trabajaron en la confección del modelo y en septiembre de 2016 publicaron Records in contexts. A conceptual model for archival description (RiC-CM). Este documento aún se encuentra en fase de borrador hasta su aprobación concluyente tras la consulta a la comunidad archivística internacional. Según sus autores, RiC-CM respeta las primicias y necesidades de los archiveros y se basa en el principio duradero de procedencia; además se centra en la separación y la interrelación de los principales componentes de la descripción archivística para 
proporcionar una base a la implementación de sistemas de descripción de archivos (Gueguen et al., 2013).

Relacionar los documentos en su contexto es la idea medular de este modelo; a partir del análisis de la procedencia se plantean nuevas dinámicas para la descripción. El principio de Origen y Procedencia, con sus dos enunciados: Respeto a los fondos y Respeto al orden natural, ha marcado la teoría y la práctica archivística durante el período moderno de esta ciencia. En este contexto, la descripción tenía como función representar intelectualmente la información del conjunto y sus partes.

\section{Records in contexts ubica a los archivos} en el camino hacia la interoperabilidad semántica descentralizada y no fundamentada en el intercambio de esquemas de metadatos

El principio de procedencia, como se entiende tradicionalmente, ha sido cuestionado por los profesionales del área, tanto desde el entendimiento teórico como en su aplicación práctica. El fundamento del respeto a los fondos da relevancia al agente que crea, acumula o conserva documentos, sin embargo no refleja íntegramente las interacciones complejas (sociales y materiales) que poseen intrínsecamente los orígenes de la documentación. Siguiendo esta idea, en RiCCM se expone que:

[...] el surgimiento de la edición colaborativa en el ambiente de redes digitales, la creación de documentos que tienen una autoría compleja, el uso de servicios de almacenamientos remotos (nube) que no son controlados completamente por los usuarios, traen problemas de propiedad y custodia. Todas esas observaciones y otras permiten llegar a la conclusión de que la procedencia es mucho más compleja que la comprensión establecida, desde hace años, de los fondos: un fondo no existe aisladamente, sino con capas de contextos interconectados de pasado, presente y futuro (ICA, 2016).

También ha sido debatido entre los profesionales el respeto al orden natural, que se refiere a que la documentación que ha producido una institución, en el archivo ha de ser estructurada de acuerdo con el orden natural que la institución le dio origen. Sin embargo, los documentos casi siempre han llegado a los archivos desordenados y los archiveros han reconstruido el origen natural a partir de la evidencia obtenida de los propios documentos; por tanto el orden original es capturado a partir de la interpretación propia de los archiveros y no de sus contextos reales de producción. Siguiendo el modelo internacional, con el trascurso del tiempo el orden de los conjuntos documentales es dinámico, cambiante y fluido y los conjuntos pueden ser registrados más de una vez (ICA, 2016).

\section{Este modelo}

"aspira tanto a reflejar las facetas del principio de procedencia, como tradicionalmente han sido entendidas y practicadas pero al mismo tiempo pretende mostrar un concepto más amplio y dinámico del entendimiento de la procedencia" (ICA, 2016).

El origen debe respetar los fondos y el orden original, aunque desde la comprensión de sus interrelaciones con las dinámicas sociales, culturales y económicas que dan origen a la documentación y su conservación. La descripción representa las complejidades y convergencias de los contextos de producción y acumulación, desmarcándose de la fisicalidad y comprendiendo la procedencia como un constante devenir y no como una acción estática e invariable.

En RiC-CM, igualmente, se expone cuáles son los propósitos de la descripción archivística en los contextos actuales de posmodernidad. El ICA (2016) propone tres roles fundamentales:

- Gestión documental;

- Preservación de documentos; y

- Reutilización de datos.

La descripción se adapta a los nuevos tiempos y a través de la representación de contenidos y contextos se contribuye a la ejecución de esos roles.

En los sistemas de gestión documental la definición de metadatos descriptivos garantiza la representación exacta del contexto, los contenidos y la estructura de los documentos, lo que asegura una gestión a corto, medio y largo plazo (ISO15489, 2016). Representar las funciones, actividades y transacciones que dan origen a la documentación también facilita el uso continuado de los registros y salvaguarda la memoria institucional, personal y colectiva, aspectos determinantes para la gestión.

RiC-CM instaura las bases para que la información archivística sea asequible, abierta y transparente $y$, con ello, garantizar la participación activa de la ciudadanía

Al pronunciarse por una descripción encauzada a la gestión, el ICA sitúa esta actividad en un lugar indispensable dentro de la gestión integrada de procesos en las organizaciones; por ende, cambia completamente la percepción técnica de la descripción, asociada especialmente con los archivos históricos. Aplicar normas y modelos para representar únicamente documentos con valor secundario es una filosofía coligada a los tiempos custodiales o modernos de la Archivística.

Según RiC-CM, las buenas prácticas descriptivas garantizan además la preservación de información digital. La preservación mantiene la integridad, fidelidad, durabilidad y legibilidad de la información a lo largo del tiempo, con independencia de las tecnologías utilizadas.

Cuando se describen entidades archivísticas mediante metadatos se viabiliza el mantenimiento de la autenticidad e integridad de los documentos. En RiC-CM se expone que:

"documentar el contexto describiéndolo es esencial para la conservación de los registros" (ICA, 2016). 
Las estrategias de preservación se enfocan en salvaguardar y mantener paquetes de datos; por consiguiente, en este medio, es importante resaltar que los metadatos descriptivos (de contexto, contenido y estructura) son determinantes para la conservación de la memoria mundial a largo plazo.

Por último, el modelo internacional hace referencia a la importancia de la descripción para la reutilización de los datos archivísticos en el entorno web. La reutilización de información está asociada al acceso, la calidad y la usabilidad de datos públicos abiertos (open data government) que posibilitan la creación de nuevos datos, contenidos, conocimientos, productos o servicios. Según un estudio realizado por investigadores de la Universidad Complutense de Madrid, las prácticas exigen que la información pública sea abierta e interoperable, es decir, que exista un modelo de información/datos que permita la colaboración entre las administraciones públicas y de éstas con los ciudadanos en beneficio de la transparencia, la interoperabilidad y la generación de nuevas actuaciones (Ramos-Simón et al., 2012).

Sobre la reutilización de datos archivísticos, en RiC-CM se señala la siguiente idea:

[...] la descripción facilita a las personas que quieren utilizar los registros documentales como evidencia para fines académicos, comerciales, personales o de otro tipo, el descubrimiento, la localización, la identificación, la recuperación, la evaluación y la comprensión de los mismos. Este uso continuo y reutilización de los registros se convierte en parte de la historia de los registros; los re-contextualiza. El uso y la reutilización generan otros registros, ampliando así la red social de información (ICA, 2016).

RiC-CM está posicionando las bases para que la información archivística sea asequible, abierta y transparente y con ello garantizar, a través del acceso de la información, la participación activa de la ciudadanía. Con este modelo se consigue reflejar un consenso con la realidad archivística internacional y convierte la descripción en un proceso eficaz para la reutilización de datos con calidad, la preservación de información digital, la gestión y la interoperabilidad de los documentos.

\section{RiC-CM refleja las facetas del principio de procedencia, pero al mismo tiempo muestra un concepto más amplio y diná- mico del mismo}

\section{Records in contexts: Entidades archivísticas}

Las entidades son el recurso de interés central en los modelos archivísticos. Una entidad, según la Comisión de Normas Españolas de Descripción Archivística (Cneda), es:

"cualquier objeto real o abstracto que existe, ha existido o puede llegar a existir" (Cneda, 2012).

En RiC-CM se han identificado, diferenciado y definido las entidades que estarán presentes en los sistemas de descripción archivísticos (tabla 1).
Tabla 1. Tipos de entidades definidas en RiC-CM

\begin{tabular}{|c|l|}
\hline \multicolumn{2}{|c|}{ Modelo conceptual internacional (RiC-CM). Entidades archivísticas } \\
\hline RiC-E1 & Documento \\
\hline RiC-E2 & Componente documental \\
\hline RiC-E3 & Conjunto documental \\
\hline RiC-E4 & Agente \\
\hline RiC-E5 & Ocupación (profesión) \\
\hline RiC-E6 & Posición \\
\hline RiC-E7 & Función \\
\hline RiC-E8 & Función abstracta \\
\hline RiC-E9 & Actividad \\
\hline RiC-E10 & Norma (mandato) \\
\hline RiC-E11 & Forma documental \\
\hline RiC-E12 & Período/fechas \\
\hline RiC-E13 & Lugar \\
\hline RiC-E14 & Concepto/asunto \\
\hline
\end{tabular}

Fuente: Elaborado a partir del ICA, 2016²

Las entidades presentadas en la tabla 1 constituyen los objetos archivísticos que serán descritos e interrelacionados en los sistemas integrados de información. El ICA, basado en sus cuatro estándares, y en otros modelos conceptuales de las ciencias de la información (cuadro 1 ) ha propuesto unas entidades que representan la realidad archivística (siguiendo una perspectiva teórica y práctica) y con ello llega a un consenso internacional.

RiC-CM contempla las entidades definidas en ISAD(G) (documento), en ISAAR(CPF) e ISDIAH (agente) y en ISDF (funciones, actividades), unificando todos los componentes del conjunto normativo. Asimismo se puede apreciar que RiCCM tiene elementos coincidentes con las FRBR, CRM y los modelos nacionales de archivos. Las entidades documento, agente, función, norma, lugar, período y concepto son denominadores comunes en los modelos conceptuales desarrollados en las ciencias de la información y constituyen entidades principales. Por ende, este modelo internacional de descripción acentúa la idea de crear un modelo semántico único que facilite la representación, la relación y la interoperabilidad de los contenidos de todas las instituciones de información en el contexto web.

Cuadro 1. Algunos modelos conceptuales para archivos, bibliotecas y museos

-Australian Government recordkeeping metadata standard.

-Technical specifications for the electronic recordkeeping metadata standard, de Nueva Zelanda.

-Finnish conceptual model for archival description.

-Modelo conceptual de descripción archivística, y Requisitos de datos básicos de las descripciones de documentos de archivo, agentes y funciones, de España.

-Requisitos funcionales para registros bibliográficos (FRBR). -Conceptual reference model (CRM), del Consejo Internacional de Museos. 
No obstante, en RiC no se incluyen entidades como manifestación, expresión, ítem, colección u objeto que fueron definidos en el Modelo Conceptual Archivístico de Finlandia, en el grupo 1 de entidades de FRBR o en el CRM, lo que demuestra que también se pueden establecer diferenciaciones en los esquemas descriptivos sin que ello dificulte la compatibilidad entre los sistemas de información.

A partir del análisis de estas entidades se percibe explícitamente la importancia del contexto para el proceso. Los objetos de información: agente, ocupación, posición, función, función abstracta, actividad, norma (legislaciones/políticas/ procedimientos) son determinantes para fijar el contexto de creación de los records y garantizar así la veracidad, integridad, autenticidad, preservación y gestión de los documentos archivísticos.

La aplicación de conceptos como web semántica, ontologías, esquemas de datos o datos abiertos entrelazados es determinante para las buenas praxis en la descripción de recursos archivísticos

En RiC-CM se definen igualmente entidades como lugar, período, concepto o asunto, entre otras determinantes para el proceso de búsqueda y recuperación de información. Estas entidades constituyen algunos de los puntos de acceso más relevantes y son, con frecuencia, los más utilizados por los usuarios para obtener los contenidos deseados.

En el modelo no se presentan directamente subtipos de estas entidades; se explica que cada una de las entidades se tratará como tipos distintos y deben ser descritas de forma diferente, aunque muchas propiedades serán compartidas. En RiC se aboga por una separación de los principales componentes de la descripción, aunque interrelacionados entre sí, pues un tratamiento diferenciado de los records, por ejemplo, va a reflejar mejor su contexto y propósito de creación.

En las normas internacionales, nacionales y los modelos conceptuales archivísticos anteriormente publicados se definen subtipos para entidades como documento, agente, función, norma o relaciones. En RiC-CM no se realizan estas categorizaciones. La concepción de nivel de organización precisando los niveles de descripción no se vislumbra en este nuevo modelo donde la jerarquía no es determinante, sino las representaciones únicas basadas en relaciones concisas entre entidades independientes que sean capaces de captar los disimiles y complejos contextos de creación.

$\operatorname{ISAD}(\mathrm{G})$ se basa en el principio de descripción multinivel y como consecuencia asume la descripción de un fondo como una entidad completa y simple, donde se describen el conjunto como un todo y sus partes, todos relacionados en la forma de jerarquía simple que presenta el modelo. En RiCCM la descripción se presenta en forma de red y no como un modelo jerárquico, permitiendo así la descripción de fondos individuales pero asumiendo la representación de las diversas capas de contextos que existen entre estos fondos y otros. Este esquema pluridimensional presenta la descrip- ción de los documentos y los conjuntos de documentos, sus interrelaciones entre sí y las interrelaciones con otras entidades. RiC reconoce el respeto a los fondos pero, además, permite trabajar con conjuntos documentales de procedencias complejas (ICA, 2016).

Aunque en el modelo no se definen subtipos para algunas entidades (RiC-E3, RiC-E4, RiC-E7, RiC-E10), posteriormente éstas son categorizadas en la definición de las propiedades. A través de la propiedad Type se concreta e tipo o subtipo de cada una. Esto demuestra que el modelo no descarta totalmente las descripciones en jerarquías aunque defiende una descripción basada en los tejidos de las entidades relacionadas.

\section{Records in contexts: Propiedades y relaciones}

Las propiedades definidas en el modelo son otro aspecto determinante para la comprensión de los nuevos enfoques descriptivos. Tal y como se ha explicado, con RiC-CM se pretende realizar una unión de ISAD(G), ISAAR(CPF), ISDF e ISDIAH. La determinación de un conjunto de propiedades que recogen, simplifican y amplían los elementos descriptivos del conjunto normativo del ICA ha demostrado la voluntad de unificar y consolidar estos estándares. El modelo aboga por representaciones únicas y precisas de cada entidad, sin embargo se delimitan varias propiedades que pueden ser compartidas.

En RiC-CM se determinan 69 propiedades para describir las 14 entidades archivísticas. En ISAD(G), ISAAR(CPF), ISDF e Isdiah se definieron un total de 117 propiedades para describir cuatro entidades (documento, agente, función e institución de custodia). La reducción en la cantidad de atributos para describir un mayor número de entidades es, sin duda, una de las fortalezas de este nuevo modelo que pretende presentar descripciones completas, pero a la vez sucintas.

\section{Los archiveros se enfrentan a un proce- so de transición en lo que respecta a la descripción}

Existen algunas propiedades (desde la RiC-P1 hasta la RiCP4) cuyo dominio de aplicación se extiende a todas las entidades del modelo. El resto de propiedades se definen de forma específica para entidades concretas. Las propiedades definidas para documentos (RiC-E1), componente documental (RiC-E2), y conjunto de documentos (RiC-E3) son próximas a los elementos de $\operatorname{ISAD}(\mathrm{G})$; las definidas para agentes (RiC-E4) contienen algunos de los elementos descriptivos de ISAAR(CPF) e ISDIAH, y las determinadas para función (RiC-E7) y actividades (RiC-E9) recogen elementos de ISDF. No obstante, RiC incluye variaciones importantes en los atributos relacionadas con los nuevos propósitos del modelo (RiC-P5 / RiC-P22: Nota de autenticidad e integridad, RiC-P8: Calidad de información, RiC-P10: Formato de codificación, RiC-P21: Estado del documento, entre otros).

Para las entidades documentos, componente documental y conjunto de documentos se especifican atributos de contenido, de formato, de lenguaje, de soporte, de gestión y 
Tabla 2. Propiedades definidas para entidades en RiC-CM ${ }^{3}$

\begin{tabular}{|c|c|c|c|}
\hline \multicolumn{4}{|c|}{$\begin{array}{l}\text { Modelo conceptual internacional } \\
\text { (RiC-CM). Propiedades para las entidades }\end{array}$} \\
\hline$\#$ & Propiedades & $\#$ & Propiedades \\
\hline RiC-P1 & Identificador global (para todas las entidades) & RiC-P39 & Información de contacto (Instituciones) \\
\hline RiC-P2 & Identificador local (para todas las entidades) & RiC-P40 & Horarios de atención (Instituciones) \\
\hline RiC-P3 & Nombre (para todas las entidades) & RiC-P41 & Facilidades de acceso para discapacitados (Instituciones) \\
\hline RiC-P4 & Nota general (para todas las entidades) & RiC-P42 & $\begin{array}{l}\text { Tipo de ocupación (abogado, profesor, personal de } \\
\text { salud, ingeniero, político...) }\end{array}$ \\
\hline RiC-P5 & Nota de autenticidad e integridad para documento & RiC-P43 & Descripción de la ocupación (funciones, actividades) \\
\hline RiC-P6 & Tipo de Contenido & RiC-P44 & Historia \\
\hline RiC-P7 & Extensión de contenido & RiC-P45 & Tipo de posición (académico, técnico, administrativo) \\
\hline RiC-P8 & Calidad de información & RiC-P46 & Descripción de la posición \\
\hline RiC-P9 & Alcance y contenido & RiC-P47 & Historia \\
\hline RiC-P10 & Formato de codificación & RiC-P48 & Tipo de función (función, sub-función) \\
\hline RiC-P11 & Lengua/Escritura & RiC-P49 & Descripción de la función \\
\hline RiC-P12 & $\begin{array}{l}\text { Tipo de medio o equipo para reproducir (computadora, audio, } \\
\text { microfilm, entre otras) (Media type) }\end{array}$ & RiC-P50 & Historia \\
\hline RiC-P13 & Requisitos técnicos de producción & RiC-P51 & Descripción de función abstracta \\
\hline RiC-P14 & Material físico en el que se representa la información (Medium) & RiC-P52 & $\begin{array}{l}\text { Tipo de actividad (actividad, proceso, acción, tarea, } \\
\text { transacción...) }\end{array}$ \\
\hline RiC-P15 & Extensión física y lógica & RiC-P53 & Descripción de la actividad \\
\hline RiC-P16 & Nota sobre características físicas & RiC-P54 & Historia \\
\hline RiC-P17 & Clasificación & RiC-P55 & $\begin{array}{l}\text { Tipo de norma o regulación (decreto, legislación, regla, } \\
\text { carta de nombramiento) }\end{array}$ \\
\hline RiC-P18 & Condiciones de acceso & RiC-P56 & Descripción de la norma o regulación. \\
\hline RiC-P19 & Condiciones de uso & RiC-P57 & Historia \\
\hline RiC-P20 & Historia & RiC-P58 & Tipo de forma documental (long playing, carta, registros) \\
\hline RiC-P21 & Estado del documento (copia, borrador, original) & RiC-P59 & Descripción \\
\hline RiC-P22 & $\begin{array}{l}\text { Nota de autenticidad e integridad para conjuntos de docu- } \\
\text { mentos }\end{array}$ & RiC-P60 & Historia \\
\hline RiC-P23 & $\begin{array}{l}\text { Tipo de conjunto documental (fondo, sección, serie, expedien- } \\
\text { te, colección...) }\end{array}$ & RiC-P61 & Tipo de fecha (únicas o en intervalos) \\
\hline RiC-P24 & Nota de ingreso & RiC-62 & Calendario (gregoriano, revolución francesa) \\
\hline RiC-P25 & Estado del ingreso & RiC-P63 & $\begin{array}{l}\text { Tipo de lugar (país, asentamiento, división administrati- } \\
\text { va, montaña, río) }\end{array}$ \\
\hline RiC-P26 & Organización & RiC-P64 & Coordenadas geográficas \\
\hline RiC-P27 & Clasificación & RiC-P65 & Dirección del lugar \\
\hline RiC-P28 & Historia & RiC-P66 & Tipo de concepto/asunto \\
\hline RiC-P29 & Extensión de contenido & RiC-P67 & Descripción del concepto/asunto \\
\hline RiC-P30 & Extensión física y lógica & RiC-P68 1 & Fecha asociada a las relaciones entre dos entidades \\
\hline RiC-P31 & Alcance y contenido & RiC-P69 & Lugar asociado a las relaciones entre dos entidades \\
\hline RiC-P32 & $\begin{array}{l}\text { Tipo de agente (grupo, institución, familia, conferencia, perso- } \\
\text { na, agente delegado, desconocido) }\end{array}$ & & \\
\hline RiC-P33 & Tipo de identidad del agente & & \\
\hline RiC-P34 & Lengua y escritura & & \\
\hline RiC-P35 & Historia del agente & & \\
\hline RiC-P36 & Género (persona) & & \\
\hline RiC-P37 & Características técnicas (Agente delegado) & & \\
\hline RiC-P38 & Servicios al público (Instituciones) & & \\
\hline
\end{tabular}

Fuente: Tabla elaborada a partir del ICA, 2016. 
usabilidad. En el texto se establece que para "RiC-E2 componente documental" se deben usar los mismos atributos que para "RiC-E1 documentos". Así mismo, se determina que para los conjuntos de documentos se pueden utilizar algunas propiedades definidas ya para los documentos, como: tipo de contenido, lenguaje de codificación, lengua/ escritura, requisitos técnicos, condiciones de acceso y uso, entre otras. Además, en caso de los tipos de conjuntos documentales pueden compartir al menos una propiedad en común, la que constituya la esencia de formación del conjunto como un todo (ICA, 2016).

\section{RiC-CM se adapta a las prácticas norma-} tivas y a los principios aceptados en el paradigma custodial pero va más allá

Existen propiedades específicas definidas para entidades diferentes que tienen una semántica similar e incluso idéntica. Se tienen como ejemplo las propiedades siguientes: RiC-P11 Lengua/Escritura y RiC-P34 Lengua/Escritura, RiCP17 Clasificación y RiC-P27 Clasificación, o las RiC-P20, RiCP28, RiC-P35, RiC-P44, RiC-P47, RiC-P50, RiC-P54, RiC-P57, y RiC-P60 referidas a "Historia". Su propósito y semántica es la misma pero el borrador del modelo conceptual las define como propiedades diferentes de acuerdo con la entidad que se esté describiendo. Posteriormente, en el desarrollo de una ontología para este modelo (RiC-O) es posible que se definan como una única propiedad.

La RiC-P Type, también, describe un mismo tipo de característica y está definida para varias entidades. El objetivo de esta propiedad es categorizar un mismo objeto de información dentro de una lista de subtipos de entidades específicas. Por ejemplo, la RiC-P23 Type para Records sets se define como: fondo, sección, serie, expediente, colección, proyecto, entre otros; la RiC-P32 Type para agente se define y ejemplifica como: personas, grupos, familias, instituciones, conferencias, agentes delegados y autoridad desconocida; la RiC-P55 Type para mandato se define como: decreto, legislación, regla, carta de nombramiento ${ }^{2}$ (véase la tabla 2 para el resto de entidades).

La semántica de la propiedad RiC-P Type no es igual para todas las entidades puesto que su alcance varía en función de la entidad que se describa. Esta propiedad enfatiza la idea de no establecer en el modelo sub-entidades sino de tratar a cada entidad como objetos independientes e interrelacionados representados en una red de conocimientos.

Las propiedades RiC-P68 Date y RiC-P69 Place no se usan como atributos de una entidad si no para describir las relaciones entre entidades, es decir, descripciones sobre descripciones. En los modelos conceptuales de Australia, Nueva Zelanda y Finlandia las relaciones sí constituyen una entidad independiente, sin embargo en el borrador de este modelo no se concibe de esta forma.

La definición de estas propiedades para la representación de objetos archivísticos en los sistemas de información constituye uno de las fortalezas de este modelo. Este esquema de elementos está basado en los trabajos normativos interna- cionales, nacionales y regionales desarrollados durante más de 20 años, además de las experiencias prácticas a través del uso de determinados softwares (Atom). Después de la publicación final de este modelo, las propiedades pueden variar pero la idea continuará encausada en determinar un número preciso de atributos que faciliten descripciones reales y veraces de las entidades archivísticas.

La ejemplificación de las relaciones entre las entidades es otro aspecto determinante en un modelo conceptual. Una relación es una asociación de cualquier tipo que se establece con una entidad definida en el modelo. La determinación de una amplia variedad de relaciones garantiza la interrelación de los contenidos de los documentos con los diversos ambientes en los que fueron producidos. Igualmente, el establecimiento de las redes relacionales facilita la exposición gráfica de los contenidos descriptivos en el entorno tecnológico y, a posteriori, garantiza el incremento de la capacidad de búsqueda de los sistemas así como la recuperación y el uso de la información. En RiC-CM se establecen 792 relaciones, cantidad que puede aumentar cuando se publique la versión final.

En el texto se muestra un conjunto de relaciones binarias simples para cada entidad, por ejemplo para el agente ("es creado por"/ "es creador de"). Muchas de estas relaciones tienen una semántica similar e incluso idéntica; esto puede ejemplificarse, también, con la relación "es miembro de" que se utiliza con las RiC-E1, RiC-E3 y RiC-E4; es decir, la denominación de una misma relación "es miembro de" puede definir tres relaciones diferentes: RiC-R18, RiC-R143, RiCR266 (véase RiC-CM, pp. 48-99). Con esta relación, los documentos pueden vincularse con conjuntos de documentos, los conjuntos de documentos con otros conjuntos de documentos y los agentes con otros agentes del tipo grupo. Esto sucede con otros tipos de relaciones definidas en el modelo.

\section{RiC-CM ofrece una nueva concepción teórica, estructural y semántica para la elaboración de sistemas de descripción adecuados a las tecnologías emergentes utilizadas para la recuperación y el acce- so a la información}

La enunciación de diversos tipos de relaciones entre las entidades permite interrelacionar mayor cantidad de información y con ello facilitar la reutilización de los datos enlazados. Varios autores consideran que la llave de la semántica entre los contenidos de los recursos de información radica en inter-relacionarlos (Sheth et al., 2002), de ahí la importancia de establecer un amplio mapa de relaciones para con posterioridad -mediante esquemas de codificación- hacerlos legibles entre computadores. La figura 1 muestra un ejemplo de descripción archivística conforme a las relaciones y las entidades definidas en RiC-CM y el diseño red que propone este modelo.

Este modelo de conceptualización ubica a los archivos en el camino hacia la interoperabilidad semántica descentralizada y no fundamentada en el intercambio de esquemas de 


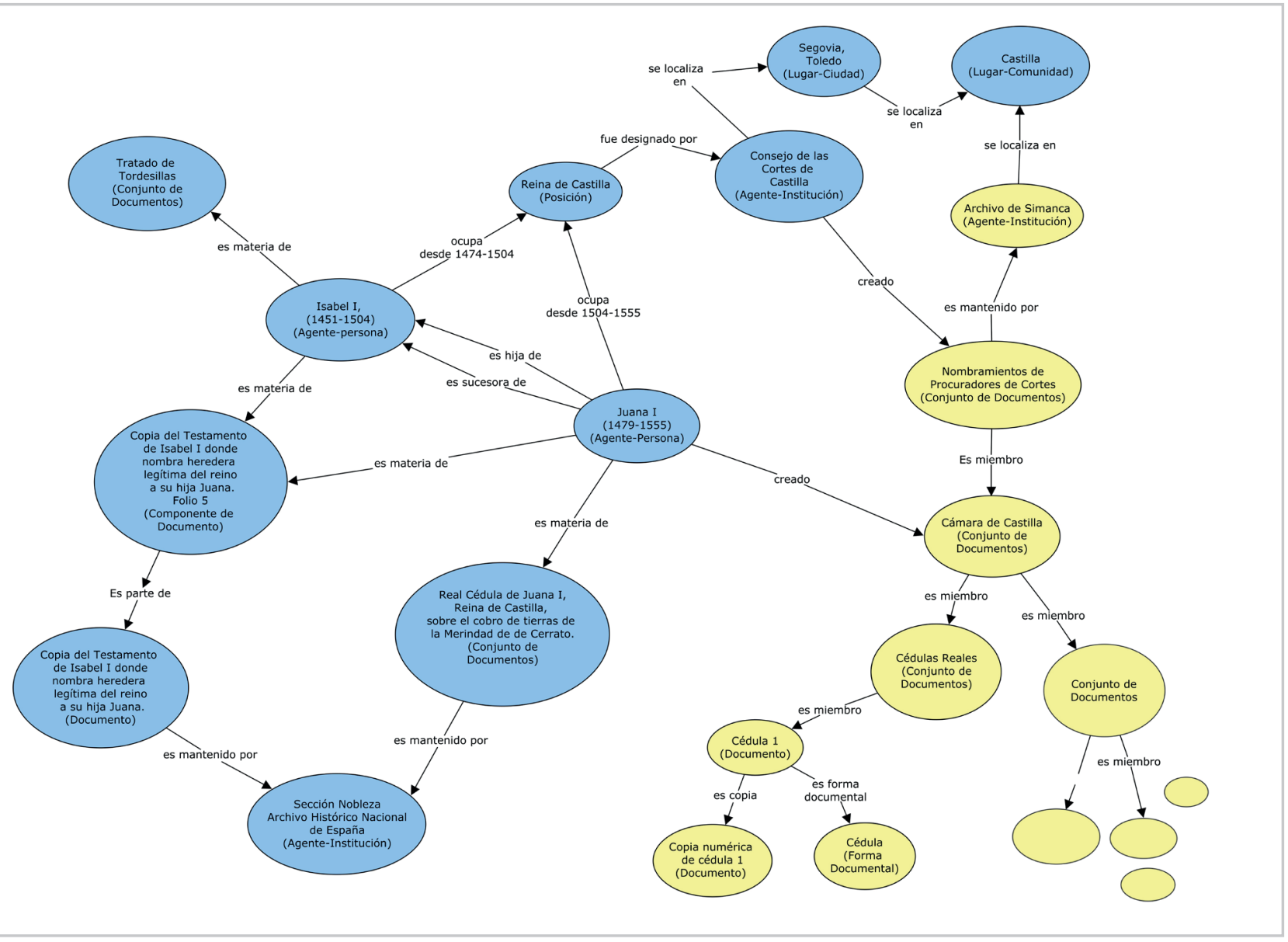

Figura 1. Ejemplo de descripción archivística a partir de RiC-CM³.

metadatos. El Expert Group on Archival Description (EGAD) está elaborando una ontología denominada $R i C-O$ para implementar el modelo RiC-CM y publicar datos archivísticos abiertos y entrelazados en la Web.

\section{Consideraciones finales}

El paradigma posmoderno en el entorno de la ciencia de la información está influyendo en el proceso de transición hacia nuevas formas de representación más alineadas con las necesidades y los enfoques actuales. La aplicación de conceptos como web semántica, ontologías (OWL), esquemas de datos (RDF) o datos abiertos entrelazados (LOD) es determinante para las buenas praxis en el proceso de descripción de recursos archivísticos. Asimismo, el uso de estas tecnologías extiende la colaboración y el intercambio de datos entre bibliotecas, museos, archivos y otras instituciones culturales.

La aplicación de RiC-CM brinda a los archivos un alto nivel de interoperabilidad semántica favoreciendo una comunicación de datos de forma directa y eficaz entre los sistemas de información. Este modelo conceptual constituye un nuevo diseño para la representación de información y la compatibilidad entre sistemas. RiC instituye una herramienta de integración y distribución de la información archivística. Con su aplicación se podrán publicar conjuntos de datos fiables, accesibles y reutilizables y se conseguirá preservar los recursos.
En la actualidad, los archiveros se enfrentan a un proceso de transición en lo que respecta a la descripción. RiC-CM se adapta a las normas internacionales, nacionales y regionales y a los principios aceptados en el paradigma custodial, pero va más allá de estas concepciones y prácticas. El modelo ofrece una nueva concepción teórica, estructural y semántica para la elaboración de sistemas de descripción adecuados con las tecnologías emergentes utilizadas en la recuperación y acceso a la información.

Records in contexts constituye el último ingrediente que se precisaba para elaborar un modelo semántico integrador. Las FRBR, Cidoc-CRM y RiC-CM pueden ser concertados y unificados con la finalidad de lograr un acceso integrado, distribuido y entrelazado del patrimonio cultural mundial. La descripción de recursos, en los tiempos posmodernos, supone un gran reto para las ciencias de la información que debe encausarse en la eliminación de las barreras conceptuales y prácticas que existen entre bibliotecas, archivos y museos en pro de un acceso abierto y libre de la información.

\section{Notas}

1. Los colores en la tabla se utilizan para diferenciar cada entidad y, posteriormente, identificar, en la siguiente tabla, las propiedades definidas por entidad.

2. El uso de los colores (en correspondencia con la tabla 1) 
es para destacar las propiedades definidas por entidades. En esta tabla no aparecen las propiedades para "RiC-E2 Componente documental" porque en el modelo se propone el uso de las mismas propiedades que para los documentos (RiC-E1).

3. Las propiedades "RiC-P68 Date" y "RiC-P69 Place" se adicionan en el modelo para describir las relaciones entre entidades.

4. Las propiedades de Type definidas en el modelo conceptual son: RiC-P23, RiC-P32, RiC-P42, RiC-P45, RiC-P48, RiCP52, RiC-P55, RiC-P58, RiC-P61, RiC-P63 y RiC-P66.

5. Este ejemplo fue construido por las autoras para este artículo, tomando como base el ejemplo que se publica en RiCCM. La diferencia de colores se establece para determinar la custodia de documentos por dos archivos: Sección Nobleza del Archivo Histórico de España y Archivo de Simancas. Los documentos "Testamento de Isabel I y Real Cédula de Juana I" se conservan realmente en la Sección Nobleza, y los documentos correspondientes a "Cámara de Castilla" se custodian en Simancas (consultado en el Portal de Archivos Españoles, Pares). El resto de datos fueron conjeturados para mostrar las relaciones que se establecen entre las entidades de RiC.

\section{Bibliografía}

Gueguen, Gretchen; Marques-da-Fonseca, Vitor-Manoel; Pitti, Daniel V.; Sibille-de Grimoüard, Claire (2013). "Hacia un modelo conceptual internacional de descripción archivística: un informe preliminar del grupo de expertos en des- cripción archivística del Consejo Internacional sobre Archivos". Métodos de información, v. 4, n. 7, pp. 155-172.

https://doi.org/10.5557/IIMEI4-N7-155172

ICA (2016). Records in contexts: A conceptual model for archival description. Consultation Draft v 0.1. International Council on Archives, Sept. 2016.

http://www.ica.org/sites/default/files/RiC-CM-0.1.pdf

ISO (2016). ISO 15489-1: Information and documentation. Records management - Part 1. April 2016.

Ministerio de Educación, Cultura y Deporte. Comisión de Normas Españolas de Descripción Archivística (2012). "Modelo conceptual de descripción archivística y requisitos de datos básicos de las descripciones de documentos de archivo, agentes y funciones". 18 junio 2012.

https://goo.gl/unJAtf

Ramos-Simón, Luis-Fernando; Arquero-Avilés, Rosario; Botezan, Iuliana; Cobo-Serrano, Silvia; Sala-Jiménez, Andrea; Sánchez-Jiménez, Rodrigo; Gastaminza, Félix del Valle (2012). "De la reutilización de la información del sector público a los portales de datos abiertos en Europa". BiD: textos universitaris de biblioteconomia i documentació, diciembre, n. 29 http://bid.ub.edu/29/pdf/ramos2.pdf

Sheth, Amit; Arpinar, I. Budak; Kashyap, Vipul (2002). Relationships at the heart of semantic web: Modeling, discovering, and exploiting complex semantic relationships. Technical report, LSDIS Lab, Computer Science, University of Georgia.

http://Isdis.cs.uga.edu/lib/download/SAK02-TM.pdf
La Fundación Biblioteca Social es una institución sin ánimo de lucro que se constituye en el año 2014. Tiene como objetivo contribuir a compensar los desequilibrios sociales apoyando proyectos que llevan a cabo las bibliotecas públicas, dirigidos a los sectores más vulnerables de la sociedad.

\section{¿Colaboras?}

fundacionbibliotecasocial.org info@fundacionbibliotecasocial.org

@Biblio_Social

FundacionBibliotecasSocial

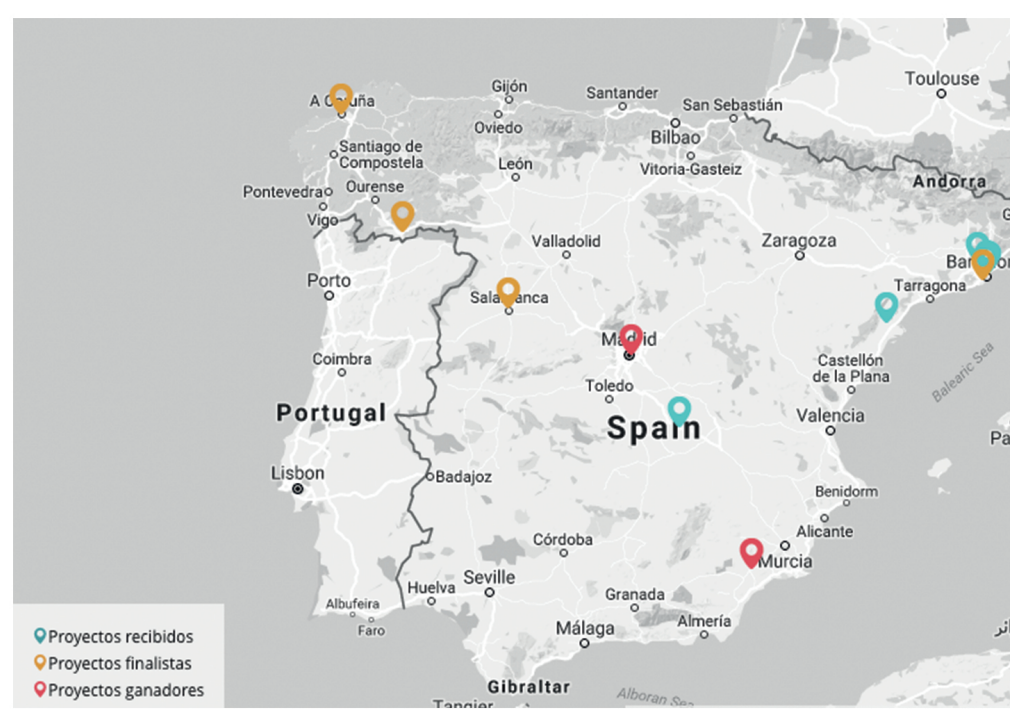

Mapa de proyectos de bibliotecas públicas para la inclusión social. 2016

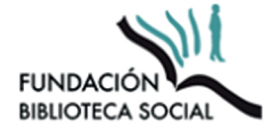

